\title{
UVA-1 cold light treatment of SLE: a double blind, placebo controlled crossover trial
}

\author{
M C A Polderman, T W J Huizinga, S Le Cessie, S Pavel
}

\begin{abstract}
Objective-Treatment of patients with systemic lupus erythematosus (SLE) often implies strong drugs with possibly serious side effects. Thus there is a need for new immunosuppressive treatments. Long wave ultraviolet A (UVA-1) cold light therapy is an anti-inflammatory, immunomodulatory treatment with a possible systemic effect and few side effects. In the current study low dose UVA-1 cold light treatment was tested to determine whether it reduces disease activity in SLE. Methods-Eleven patients with SLE were treated with UVA-1 cold light treatment and a placebo light treatment in a double blind, placebo controlled, crossover study. In two consecutive 12 week periods the patients were treated in the first three weeks with UVA-1 and placebo treatment or vice versa. The primary variables were the SLE Disease Activity Index (SLEDAI) and SLE Activity Measure (SLAM).
\end{abstract}

Results-The mean SLAM and SLEDAI showed a significant decrease of $30.4 \%$ $(p=0.0005)$ and $37.9 \%(p=0.016)$ respectively after three weeks of UVA-1 and a non-significant decline of $9.3 \%(p=0.43)$ and $12.2 \% \quad(p=0.54)$ respectively after three weeks of placebo treatment. In this small trial the difference in reduction of the disease activity indices during UVA-1 compared with during placebo treatment failed to reach the conventional border of significance $(p=0.07)$. The total score of quality of life measure RAND-36 did not improve significantly, but the subscore for vitality did improve.

Conclusion-Low dose UVA-1 cold light treatment was strongly suggestive of lowering disease activity in this double blind placebo controlled study, and no side effects occurred.

(Ann Rheum Dis 2001;60:112-115)

Current treatment of systemic lupus erythematosus (SLE) comprises non-steroidal antiinflammatory drugs (NSAIDs), antimalarial drugs, prednisone, azathioprine, cyclophosphamide, chlorambucil, and methotrexate. These are drugs with potential side effects. Thus there is a need for alternative immunosuppressive treatments. Long wave ultraviolet A (UVA-1) cold light therapy is an immunosuppressive treatment ${ }^{12}$ with proven efficacy in patients with atopic dermatitis. ${ }^{3}$ The main short term side effects are a little sunburn and slight xerosis cutis. Although animal experiments suggest that UVA-1 is less carcinogenic than UVA-2 and UVB, ${ }^{4}$ the severity of long term side effects, like carcinogenicity and aging, is not yet clear. Compared with UVB $(280-320 \mathrm{~nm})$ and UVA-2 (320-340 nm), UVA-1 (340-400 nm) penetrates deeper into the skin, as far as the deeper layers of the dermis. Because of that deeper penetration the immunosuppressive and anti-inflammatory effects of UVA-1 are thought to be moderately systemic.

For a long time exposure to sunlight has been associated with exacerbation of SLE. ${ }^{56}$ Approximately $45 \%$ of patients with SLE are known to have photosensitivity. ${ }^{7}$ After exposure to sunlight patients show persistent erythema, erythematous papules, or papulovesicles. Mainly UVB and, to a lesser extend UVA, are held responsible for the signs of photosensitivity occurring. ${ }^{8}$ Accordingly, the first reports on the beneficial effects of long wave UVA-1 in patients with SLE were unexpected.

In 1987 McGrath Jr et al described the favourable effect of UVA on SLE activity. Survival was prolonged only in irradiated mice in the New Zealand black/New Zealand white mouse model of SLE. Irradiated mice, compared with those not irradiated, had decreased anti-dsDNA levels and decreased spleen size at necropsy. Irradiation comprised wavelengths predominantly in the UVA range (320-400 nm). ${ }^{9}$ Later, these authors also reported that low dose UVA-1 induced decreases of clinical disease activity, doses of systemic steroids, and autoantibodies in humans and improved disease activity scores during maintenance treatment when patients were irradiated twice a week for eight months after the initial three week treatment period. ${ }^{10}{ }^{11}$ In 1993 Sönnichsen et al published a case report about the successful treatment of a patient with subacute cutaneous lupus erythematosus with UVA- $1 .^{12}$

As UVA-1 irradiation may be promising in the treatment of SLE and as studies to determine the efficacy of UVA-1 in the treatment of SLE have been carried out by one research group only, we treated 11 patients with SLE in a double blind, placebo controlled, crossover study to compare results and establish a basis for further clinical and laboratory investigation.

\section{Patients and methods}

\section{PATIENTS}

Eleven patients with mild to moderate SLE were included in this prospective study (table 1). Patients (one male, 10 female) were recruited from the SLE outpatients' clinic of the rheumatology department. Their mean age was 38.1 years (range 18-56, median 35). Nine 
Table 1 Patients' characteristics

\begin{tabular}{|c|c|c|c|c|c|c|c|c|c|c|c|}
\hline $\begin{array}{l}\text { Patient } \\
\text { No }\end{array}$ & ${ }^{t} \mathrm{Age}$ & Sex & $\begin{array}{l}\text { Skin } \\
\text { colour }\end{array}$ & $\begin{array}{l}\text { Disease } \\
\text { (years) }\end{array}$ & $\begin{array}{l}\text { SLEDAI } \\
\text { at start } \\
\text { study }\end{array}$ & $\begin{array}{l}\text { SLAM at } \\
\text { start study }\end{array}$ & $\begin{array}{l}\text { SLEDAI } \\
\text { duringt } \\
\text { UVA-1 }\end{array}$ & $\begin{array}{l}\text { SLEDAI } \\
\text { duringt } \\
\text { placebo }\end{array}$ & $\begin{array}{l}\text { SLAM } \\
\text { duringt } \\
\text { UVA-1 }\end{array}$ & $\begin{array}{l}\text { SLAM } \\
\text { duringt } \\
\text { placebo }\end{array}$ & Drugs \\
\hline 1 & 35.0 & $\mathrm{~F}$ & White & 6 & 17 & 9 & -1 & -0.5 & -3 & -2 & Plaquenil $1 \times 200 \mathrm{mg}$ \\
\hline 2 & 29.2 & $\mathrm{~F}$ & White & 7 & 12 & 12 & -9 & +2 & -1 & +1 & Plaquenil $2 \times 200 \mathrm{mg}$, diclofenac $2 \times 75 \mathrm{mg}$ \\
\hline 3 & 27.5 & $\mathrm{~F}$ & White & 6 & 13 & 17 & -4 & -3 & -6 & -3 & Ibuprofen $3 \times 400 \mathrm{mg}$ \\
\hline 4 & 48.6 & $\mathrm{~F}$ & Dark & 3 & 12 & 15 & -10 & 0 & -2 & -6 & Plaquenil $1 \times 200 \mathrm{mg}$, prednisone $1 \times 10 \mathrm{mg}$, diclofenac $2 \times 50 \mathrm{mg}$ \\
\hline 5 & 30.9 & $\mathrm{~F}$ & White & 8 & 16 & 13 & -2 & -4 & -6 & +8 & Plaquenil $2 \times 200 \mathrm{mg}$, prednisone $1 \times 5 \mathrm{mg}$, naproxen $250 \mathrm{mg} /$ week \\
\hline 6 & 18.4 & $\mathrm{~F}$ & White & 2 & 7 & 12 & +6 & +1 & -1 & +2 & Plaquenil $1 \times 200 \mathrm{mg}$, prednisone $1 \times 5 \mathrm{mg}$, ibuprofen $2 \times 800 \mathrm{mg}$ \\
\hline 7 & 54.8 & $\mathrm{~F}$ & Dark & 5 & 26 & 15 & -19 & +6 & -3 & 0 & Prednisone $1 \times 15 \mathrm{mg}$ \\
\hline 8 & 34.9 & $\mathrm{~F}$ & White & 19 & 14 & 16 & -2 & -2 & -8 & 0 & Plaquenil $1 \times 200 \mathrm{mg}$ \\
\hline 9 & 41.4 & $\mathrm{~F}$ & White & 8 & 10 & 13 & -4 & -6 & -7 & -5 & Prednisone $1 \times 7 \mathrm{mg}$, naproxen $3 \times 250 \mathrm{mg}$ \\
\hline $10^{\star}$ & 42.0 & $\mathrm{~F}$ & White & 17 & 12 & 15 & -9 & 0 & -4 & +1 & Plaquenil $3 \times 200 \mathrm{mg}$, prednisone $1 \times 5 \mathrm{mg}$, ibuprofen $2 \times 400 \mathrm{mg}$ \\
\hline $11^{\star}$ & 56.2 & $\mathrm{M}$ & White & 5 & 20 & 17 & -7 & -8 & -4 & -9 & Arthrotec $1 \times 75 \mathrm{mg}$ \\
\hline
\end{tabular}

*Patients who recieved placebo treatment first.

†Decrease $(-)$ and increase ( + ) of SLAM and SLEDAI score during UVA-1 and placebo treatment.

patients were white subjects, one was Surinam creole, and one was Indonesian. At entry their disease had a mean duration of 7.8 years (range 2-19, median 6). All patients fulfilled four or more American College of Rheumatology criteria for the diagnosis SLE and an SLE Disease Activity Index $\left(\right.$ SLEDAI $\left.^{13}\right)$ of at least four. Patients were not allowed to change their drugs two months before entry. During the study, changes in drugs (except for NSAIDs) could only be made by the rheumatologist.

\section{IRRADIATION EQUIPMENT}

For UVA-1 irradiation the Photomed 250000 (Photomed Medizintechnik GmbH Vertrieb Deutschland, Gehrden, Germany) was used. It emits photons with wavelengths longer than $340 \mathrm{~nm}$. The instrument is equipped with a filter system that eliminates all infrared radiation, which significantly reduces heat production and increases comfort for the patients. Owing to these filters, the ventilation system that provides the patient with a cool breeze, and the blue colour of the light, Photomed UVA-1 treatment is also called UVA-1 cold light treatment.

The placebo treatment comprised TL light tubes that could be placed under the UVA-1 light tubes. In this way patients used the same cabin for both treatments. To match the blue colour of the UVA-1 treatment, blue plastic covered the frame with the TL light tubes. Patients could recognise differences between the lamps but they did not know which was the supposedly effective treatment. During both treatments patients wore protective eyewear.

TREATMENT SCHEDULE

The study had a double blind, placebo controlled, crossover design. During two consecutive 12 week periods patients were treated in the first three weeks. The following nine weeks served as a wash out period. Patients were randomly allocated to group A $(n=9)$ or group B $(n=2)$ by an independent person. Irradiation consisted of total body irradiation with $6 \mathrm{~J} / \mathrm{cm}^{2}$, five days a week for three weeks or an equivalent time of exposure (3 minutes, 20 seconds) to placebo light. Group A was treated with UVA-1 for the first three weeks and was crossed over to be treated with the placebo light treatment in the second treatment period. Group B was treated with both UVA-1 and placebo light treatment in reverse succession.
Irradiation was carried out during the winter months to minimise concomitant exposure to natural sunlight. Variables were evaluated every three weeks by the doctor until nine weeks after the last three week treatment period. Both the doctor and the patients were blinded to the treatment throughout the study.

Our primary measures were two systems for clinical assessment of SLE disease activity, the SLE Disease Activity Index (SLEDAI ${ }^{13}$ ) and the SLE Activity Measure (SLAM). ${ }^{14}$ The SLEDAI consists of 19 items representing nine organ systems. Each item is rated as present or absent. The SLAM includes 24 clinical manifestations for nine organ systems and eight laboratory variables to evaluate organs that cannot otherwise be assessed. All items are scored as 0 to 2 or 0 to 3 according to their severity. Two $100 \mathrm{~mm}$ visual analogue scales (VAS) accompany the SLAM score to measure the patient's and doctor's subjective ratings of disease activity. We decided to use the SLEDAI score because it discriminates single disease activity states among subjects well and completion costs little time. We included the SLAM score because it detects a treatment effect more sensitively. ${ }^{14}$ Furthermore, drugs were monitored and the patients filled in a validated quality of life questionnaire, the RAND-36, ${ }^{15}$ at each control visit. This quality of life questionnaire was rated in total as well as in separate scores for different features of quality of life: physical, social, and mental functioning, pain, vitality, and change in state of health. Apart from evaluation of clinical variables, titres of antibodies to SSA, SSB, Sm, and RNP were determined as well as antinuclear antibodies and anti-dsDNA. Furthermore, a complete blood count, an erythrocyte sedimentation rate (ESR), and urine analysis were done every three weeks.

\section{STATISTICAL METHODS}

McGrath found a $39 \%$ decrease ( $=6$ points, SD 4.295, $\mathrm{p}<0.005$ ) of disease activity scores in 10 patients who were treated with UVA- $1 .^{11}$ To detect a decrease of six points when treated with UVA-1 and 0 points when treated with placebo (SD 4.295) with a power of $80 \%$ ( $\alpha=0.05$, two sided tests) 11 patients were needed. A paired Wilcoxon test was used to determine changes in clinical and laboratory variables before and after UVA-1 and placebo irradiation and to determine significant differ- 


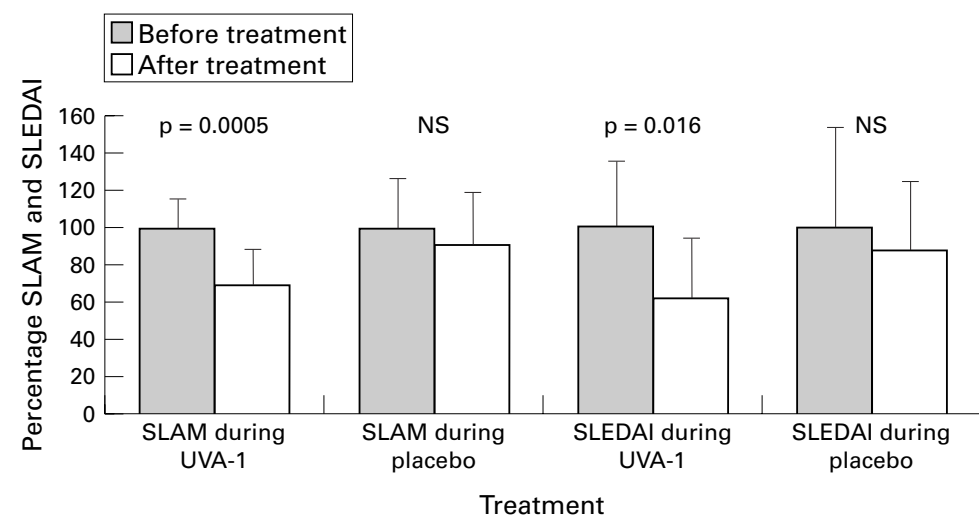

Figure 1 SLAM and SLEDAI showed statistically significant improvement during UVA-1. Improvement of these variables during placebo treatment was not statistically significant. showed significant improvement after UVA-1 cold light treatment in comparison with placebo treatment $(\mathrm{p}=0.04$ and 0.03 respectively). These results were mainly due to improvement of rash $(p=0.08)$ and Raynaud's phenomenon $(p=0.06)$. Improvements in the other seven organ systems were not statistically significant.

Also, the RAND-36 subscore for vitality improved more after UVA-1 ( -15.91 points, from 33.64 to $49.55,95 \%$ CI -29.58 to -2.24 ) than after placebo treatment (2.27 points, from 47.27 to $45.00,95 \%$ CI -8.60 to 13.14 ) $(\mathrm{p}=0.03)$. Changes in the RAND-36 total score and in the remaining subscores after UVA-1 were not statistically significantly different from changes in these scores after placebo treatment.

Four patients were anti-SSA positive. In these four patients a mean decrease of anti-SSA antibody titres of $2.75 \mathrm{U} / \mathrm{ml}$ was found after UVA-1 (from 87 to $84.25,95 \%$ CI $3.25 \times 10^{-2}$ to 5.47 ) and a very small mean increase of $0.25 \mathrm{U} / \mathrm{ml}$ after placebo treatment (from 83 to $83.25,95 \% \mathrm{CI}-3.26$ to 2.76 ) was seen; the difference was not significant $(p=0.06)$, however. Seroconversion from a positive to a negative anti-SSA status did not occur. Anti-dsDNA antibody status did not differ significantly throughout the study. Changes in the doctor's and patient's VAS and changes in the complete blood count, the ESR, and the urine analysis after UVA-1 were not different from after placebo treatment. No changes in drugs were made. None of the evaluated variables showed a period or carry over effect.

Despite the fact that five of the 11 patients were known occasionally to be photosensitive, no signs of photosensitivity or other side effects occurred during UVA-1 or placebo treatment. placebo treatment, the SLAM did not decrease significantly more after UVA-1 than after placebo treatment (mean -2.91, 95\% CI -6.39 to $0.57, \mathrm{p}=0.07)$. Similarly, the SLEDAI decreased by $37.9 \%$ ( 5.55 points, $95 \%$ CI 1.24 to 9.85 ) after UVA-1 treatment and $12.2 \%$ (1.32 points, 95\% CI -1.29 to 3.93 ) after placebo treatment-that is, from 14.64 $(=100 \%, \quad$ SD $5.12=34.97 \%)$ to 9.09 $(=62.09 \%$, SD $4.78=32.65 \%)$ after UVA-1 $(\mathrm{p}=0.016)$ and from $10.82(=100 \%, \mathrm{SD}$ $5.78=53.41 \%) \quad$ to $9.50 \quad(=87.8 \%, \quad \mathrm{SD}$ $3.93=36.32 \%)$ after placebo treatment $(p=0.54)$ (fig 1). Again, the difference between decrease of SLEDAI after UVA-1 and after placebo was not significant (mean $-4.23,95 \%$ CI -10.11 to $1.65, \mathrm{p}=0.07)$. Until six weeks after UVA-1 the decrease of SLAM and SLEDAI was significant compared with the SLAM and SLEDAI scores before UVA-1 treatment. Thus the clinical effect of UVA-1 lasted for six to nine weeks. SLAM and SLEDAI scores did not show significant decreases immediately after, three, six, or nine weeks after placebo treatment.

The nine organ systems of the SLAM score were also evaluated separately. The score of integument (oral ulcers + cutaneous rash + vasculitis + alopecia) and the cardiovascular score (Raynaud + hypertension + carditis)

\section{Discussion}

Improvement of SLAM and SLEDAI during UVA-1 treatment was significant, whereas improvement of both scores during placebo was not. Although the small number of patients resulted in a p value of 0.07 , we suggest that the better improvement of the SLAM and SLEDAI during UVA-1 compared with during placebo treatment has obvious clinical importance. We make this suggestion especially because UVA-1 has few short term side effects and certainly the side effect profile is better than that of most of the alternative treatments for SLE. Apart from improvement of SLAM and SLEDAI scores, which contain both objective and subjective variables, objective serological monitoring of disease activity by evaluating titres of anti-SSA showed an obvious trend of improvement during UVA-1 treatment in the four anti-SSA positive patients included.

The statistically significant improvement of the integument and cardiovascular subscores of the SLAM and of the vitality subscore of the RAND-36 quality of life index should be interpreted with some caution. It should be kept in mind that testing of subscores increases the risk 
of statistical significance by chance. One could correct by Bonferroni correction, though this method is considered to be conservative.

In a double blind, placebo controlled, crossover design McGrath et al treated 26 patients with SLE with less favourable results. ${ }^{16}$ After UVA-1 treatment, group A showed a significant 1.7 point decrease of the SLAM from $8.4(2.9)$ to $6.7(1.9)(\mathrm{p}<0.05)$. Decrease of the SLAM in group B after UVA-1 was not statistically significant. The lack of wash out periods in their study risked carry over effects. Furthermore, the authors did not evaluate placebo effects by comparing the changes in SLAM after UVA-1 with the changes in SLAM after placebo treatment. In an uncontrolled study McGrath treated 10 patients with $6 \mathrm{~J} / \mathrm{cm}^{2}$ UVA-1 (five times a week for three weeks). ${ }^{11}$ The treatment resulted in improvement of various clinical measures. However, these variables were not combined in a commonly used disease activity scoring system and were consequently not easily comparable with our results. Furthermore, a different type of UVA-1 lamp was used.

The working mechanisms of UVA-1 are largely unknown. In the treatment of atopic dermatitis UVA-1 light is used in much higher doses. ${ }^{3}$ Apoptosis of certain $\mathrm{T}$ cell populations, resulting from singlet oxygen generation, is believed to play a part in this therapeutic effect. Owing to the apparent risk of photosensitivity in patients with SLE, we used a very low dose of UVA-1. No signs of photosensitivity occurred in any of our patients. Symptoms of photosensitivity are reported to occur in patients with SLE when irradiated with UVA doses higher than $20 \mathrm{~J} / \mathrm{cm}^{2}, 8$ and thus a UVA-1 dose higher than $6 \mathrm{~J} / \mathrm{cm}^{2}$ might result in a better outcome. Also, it is not known how long the clinical effect of UVA-1 in patients with SLE lasts once the treatment is stopped. In our trial the effect lasted for six to nine weeks. A maintenance treatment of one or two irradiations a week might possibly prolong clinical effectivity.

The working mechanism of UVA-1, the effect of a higher dose of UVA-1, and the effect of maintenance UVA-1 treatment in patients with SLE are currently under investigation.

We thank the Dutch League against Rheumatism for financing this research project and Mrs M Kompas for analysis of the autoantibody titres.

1 Grewe M, Gyufko K, Krutmann J. Interleukin-10 production by cultured human keratinocytes: regulation by ultration by cultured human keratinocytes: regulation by ultra-
violet B and A1 radiation. J Invest Dermatol 1995;104:3-6. violet B and A1 radiation. J Invest Dermatol 1995;104:3-6.
Krutmann J. Ultraviolet A1 radiation-induced immunomodulation: high-dose UVA-1 therapy of atopic dermatitis. In: Krutmann J, Elmets G, eds. Photoimmunology. Oxford: Blackwell Science, 1995:246-56.

3 Krutmann J, Diepgen TL, Luger TA, Grabbe S, Meffert H, Sönnichsen N, et al. High-dose UVA1 therapy for atopic dermatitis: results of a multicenter trial. J Am Acad dermatitis: results of a mul

4 Gruijl FD, Sterenborg H, Forbes P, Davies R, Cole C, Kelfkens $\mathrm{G}$. Wavelength dependence of skin cancer induction by ultraviolet irradiation of albino hairless mice. Cancer Res 1993;53:53-60.

5 Feit $\mathrm{H}$. Light as exciting agent in lupus erythematosus and other dermatoses. Journal of the Medical Society of New Jersey $1927 ; 24: 226-8$.

6 Rasch C. Effect of light on skin diseases. Proc R Soc Med 1926;20:1-20.

7 Cervera R, Khamashta MA, Fong J. Systemic lupus erythematosus: clinical and immunologic patterns of disease expression in a cohort of 1,000 patients. Medicine (Baltimore) 1993;72:113-24.

8 Hasan T, Nyberg F, Stephansson E, Puska P, Häkkinen M, Sarna S, et al. Photosensitivity in lupus erythematosus, UV photoprovocation results compared with history of photosensitivity and clinical findings. Br J Dermatol 1997;136: 699-705.

9 McGrath Jr H, Bak E, Michalski JP. Ultraviolet-A light prolongs survival and improves immune function in (New Zealand black $\times$ New Zealand white) F1 hybrid mice. Arthritis Rheum 1987;30:557-61.

10 McGrath Jr H. Prospects for UV-A1 therapy as a treatment modality in cutaneous and systemic LE. Lupus 1997;6: 209-17.

11 McGrath Jr H. Ultraviolet-A1 irradiation decreases clinical disease activity and autoantibodies in patients with disease activity and autoantibodies in patients with 12:129-35.

12 Sönnichsen N, Meffert H, Kunzelmann V, Audring H. UV-A-1-Therapie bei subakut kutanem Lupus erythematodes. Hautarzt 1993;44:723-5.

13 Bombardier C, Galdman D, Urowitz M, Caron D, Chang C. Derivation of the SLEDAI. A disease activity index for lupus patients. Arthritis Rheum 1992;35:630-40.

14 Liang MH, Socher SA, Larson MG, Schur PH. Reliability and validity of six systems for the clinical assessment of disand validity of six systems for the clinical assessment of disease activity in systemic

15 Ware JE, Sherbourne CD. The RAND-36 Short-form Health Status Survey: I. Conceptual framework and item selection. Med Care 1992;30:473-81.

16 McGrath Jr H, Martinez-Osuna P, Lee FA. Ultraviolet-A (340-400 nm) irradiation therapy in systemic lupus erythematosus. Lupus 1996;5:269-74. 